\title{
Chemical Ordering in Bimetallic FeCo Nanoparticles : from a direct chemical synthesis to application as efficient high frequency magnetic material
}

Cyril Garnero, ${ }^{1}$ Mathieu Lepesant, ${ }^{1}$ Cécile Garcia-Marcelot, ${ }^{1,2}$ Yooleemi Shin, ${ }^{3,4}$ Christian Meny, ${ }^{3}$ Pierre Farger, ${ }^{1-2}$ Bénédicte Warot-Fonrose, ${ }^{2}$ Raul Arenal, ${ }^{5,6,7}$ Guillaume Viau, ${ }^{1}$ Katerina Soulantica, ${ }^{1}$ Pierre Fau, ${ }^{8}$ Patrick Poveda, ${ }^{9}$ Lise-Marie Lacroix, ${ }^{1 *}$ Bruno Chaudret $^{1}$

1. Université de Toulouse, UMR 5215 INSA, CNRS, UPS, Laboratoire de Physique et Chimie des Nano-Objets, 135 avenue de Rangueil F-31077 Toulouse cedex 4, France

2. Centre d'Elaboration de Matériaux et d'Etudes Structurales, CEMES-CNRS, 29 rue Jeanne Marvig, B.P. 94347, 31055 Toulouse, France

3. Institut de Physique et Chimie des Matériaux de Strasbourg, Université de Strasbourg, CNRS, UMR 7504, 23 rue du Loess, 67034 Strasbourg, France

4. Department of Physics, CNRS-Ewha International Research Center, Ewha Womans University, Seoul 120-750, South Korea

5. Instituto de Nanociencia de Aragon (INA), Universidad de Zaragoza, Calle Mariano Esquillor, 50018 Zaragoza, Spain

6. ARAID, 50018 Zaragoza, Spain

7. Instituto de Ciencias de Materiales de Aragon, CSIC-U. de Zaragoza, Calle Pedro Cerbuna 12, 50009 Zaragoza, Spain

8. Laboratoire de Chimie de Coordination, UPR 8241, 205 route de Narbonne, 31400 Toulouse, France

9. ST Microelectronics Tours, 10 rue Thalès de Milet, CS 97155, 37071 Tours Cedex 2, France

Corresponding author: Imlacroi@insa-toulouse.fr, Tel: +33567048833, Fax: +33561559697

Abstract : Single crystalline FeCo nanoparticles with tunable size and shape were prepared by co-decomposing two metal-amide precursors under mild conditions. The nature of the ligands introduced in this organometallic synthesis drastically affects the reactivity of the precursors and thus the chemical distribution within the nanoparticles. The presence of the B2 short-range order was evidenced in FeCo nanoparticles prepared in presence of $\mathrm{HDAHCl}$ ligands, combining ${ }^{57} \mathrm{Fe}$ Mössbauer, zero field ${ }^{59} \mathrm{Co}$ Ferromagnetic Nuclear Resonance (FNR) and X-ray diffraction studies. This is the first time that the B2 structure is directly formed during synthesis without the need of any annealing step. The as-prepared nanoparticles exhibit magnetic properties comparable with the ones of the bulk (Ms $\left.=226 \mathrm{Am}^{2} \cdot \mathrm{kg}-1\right)$. Composite magnetic materials prepared from these FeCo nanoparticles led to a successful 
proof-of-concept of integration on inductor-based filters $(27 \%$ enhancement of the inductance value at $100 \mathrm{MHz}$ ).

Key words: Magnetic nanoparticles, Zero field ${ }^{58}$ Co FNR, Mössbauer spectroscopy, composite materials, inductor-based filter

TOC :

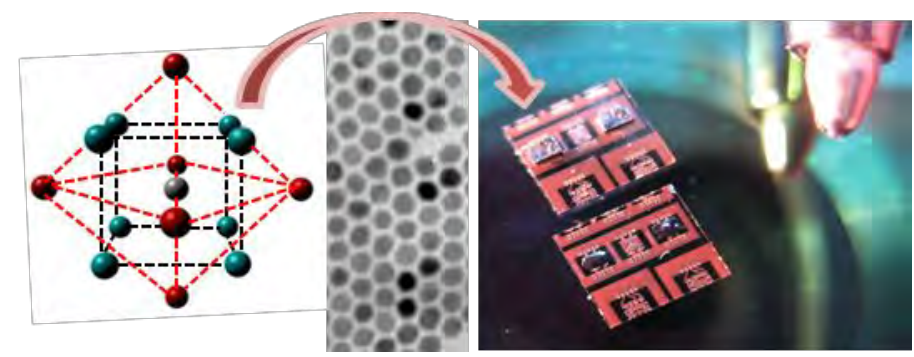


The needs for telecommunications have considerably increased in the past decade, requiring high performance, low power consumption and low cost radiofrequency circuits to support the huge expansion of local exchanges such as video on demand, interconnections with mobile phone applications or shared storage. Among the different passive components present in these circuits, integrated planar inductors play a key role to properly filter the signal of interest while rejecting the surrounding electromagnetic noise up to the $\mathrm{GHz}$, which is the range of frequency commonly used for cellular phone networks worldwide. The efficiency of these inductors, evaluated by the value of their inductance, should be constantly increased. However, geometrical features, such as number of spires and diameter of the wires, can no longer be improved without leading to larger inductors. Thus, alternatives are actively sought, the most promising one consisting in improving the environment permeability by embedding the inductors in a soft ferromagnetic material. ${ }^{1} \mathrm{An}$ alternative to the magnetic thin films commonly used consists in preparing composite materials composed of optimized magnetic nanoparticles embedded in an insulating matrix. ${ }^{2}$ Thanks to the development of liquid-phase synthesis during the past two decades, various magnetic nanoparticles (NPs) of controlled sizes and shapes were synthesized, ${ }^{3}$ opening great perspectives for RF filters, as well as answering to the increasing demand in magnetic data storage $^{4,5}$ and in chemical, ${ }^{6}$ environmental ${ }^{7}$ and biomedical applications. ${ }^{8}$ Single crystalline Fe, Co or Ni NPs have been obtained with a nanometric size control, ${ }^{9}$ and shapes ranging from spheres to cubes, rods or wires, allowing in turn a fine control of their physical properties. ${ }^{3}$ Among the different magnetic materials, the FeCo alloy constitutes the material of primary choice for magnetic inductors since it combines the highest saturation magnetization $\left(\mathrm{MS}_{\mathrm{S}} \mathrm{Fe}_{50} \mathrm{CO}_{50}=235 \mathrm{~A} \cdot \mathrm{m}^{2} \cdot \mathrm{kg}^{-1}\right)$ with a low anisotropy constant $\left(\mathrm{K} \mathrm{Fe}_{50} \mathrm{CO}_{50}=\right.$ $\left.1,5.10^{4} \mathrm{~J} \cdot \mathrm{m}^{-3}\right),{ }^{10}$ allowing high frequency applications. However, such bimetallic alloy NPs are highly challenging to synthesize. Segregation issues are often encountered between the two components, leading to core-shell, onions or dumbbell NPs. ${ }^{11}$

Despite important efforts to develop liquid phase syntheses, including thermal decomposition, ${ }^{12}$ polyol process, ${ }^{13,14}$ hydrothermal synthesis ${ }^{15-17}$ and sonochemical method, ${ }^{18,19}$ crystalline FeCo nanoparticles (NPs) could rarely be achieved directly. Indeed, the difference of reactivity of the precursors and the mild temperature of reaction used lead to poorly crystallized nanoparticles with inhomogeneous chemical structure, which therefore exhibit a reduced saturation magnetization. A high temperature annealing process 
is then mandatory to achieve the desired bulk magnetic properties, but at the expense of the size and shape control of the nanoparticles due to sintering.

Our group has previously reported the synthesis of $15 \mathrm{~nm}$ FeCo NPs, which self-organize into millimeter supercrystals, by the co-decomposition of $\mathrm{Fe}(\mathrm{CO})_{5}$ and $\mathrm{Co}\left(\eta^{3}-\mathrm{C}_{8} \mathrm{H}_{13}\right)\left(\eta^{4}-\mathrm{C}_{8} \mathrm{H}_{12}\right)$ under $\mathrm{H}_{2} \cdot{ }^{20-22}$ The particles exhibited however, a magnetization limited to $160 \mathrm{~A} \cdot \mathrm{m}^{2} . \mathrm{kg}^{-1}$ due to a chemically inhomogeneous structure and a partial carburation, which is likely resulting from the presence of $\mathrm{CO}$ in the iron precursor. ${ }^{23}$ Thanks to a fine optimization of the organometallic precursors used, we report here the synthesis of highly crystalline FeCo nanoparticles with bulk magnetic properties. Depending on the nature of the ligands used, the structure of the NPs could be varied from a radial composition gradient configuration to the chemically ordered $B_{2}$ phase, as revealed by the combined ${ }^{57} \mathrm{Fe}$ Mossbauer, zero field ${ }^{59}$ Co Ferromagnetic Nuclear Resonance (FNR) and XRD studies. The efficiency of a highly magnetic composite material, prepared with optimized FeCo NPs immersed in epoxy resin, was finally tested onto commercial inductors, leading to a $27 \%$ improvement of the properties over a broad frequency range. 


\section{Results and discussion}

FeCo nanoparticles (NPs) were obtained by reacting $\left\{\mathrm{Fe}\left[\mathrm{N}\left(\mathrm{SiMe}_{3}\right)_{2}\right]_{2}\right\}_{2}\left(\mathrm{Me}=\mathrm{CH}_{3}\right)$ and $\left\{\mathrm{Co}\left[\mathrm{N}\left(\mathrm{SiMe}_{3}\right)_{2}\right]_{2} \mathrm{THF}\right\}\left(\mathrm{THF}=\right.$ tetrahydrofuran) at $150^{\circ} \mathrm{C}$ with $\mathrm{H}_{2}$. Depending on the nature and the concentration of the added ligands, nanoparticles with a mean size varying from 1.5 to $90 \mathrm{~nm}$ were obtained (Figure S1). Figures 1a and S2 show transmission electron microscopy images (TEM) of nanocubes, with a mean size of $8.3 \pm 0.9 \mathrm{~nm}$, synthesized after $48 \mathrm{~h}$ of reaction in presence of palmitic acid and hexadecylamine in a 3:4 ratio. These particles will be referred hereinafter as FeCo-PA NPs. Replacing palmitic acid by hexadecylammonium chloride leads to NPs with a mean diameter of $11.4 \pm 0.7 \mathrm{~nm}$, referred as FeCo-HDAHCI NPs (Figures 1b, S3-4). The reaction kinetic being fastened in presence of HDAHCl ligands, the reaction time could be shortened to $24 \mathrm{~h}$ (Figures S5-6).

Both types of particles are highly crystalline and exhibit the expected bcc structure as revealed by high resolution transmission electron microscopy (HRTEM, Figures 1c-d) and Xray diffraction (XRD, Figure S7). The crystallite sizes could be extracted using Rietveld refinement and are respectively of $7.1 \mathrm{~nm}$ and $9.9 \mathrm{~nm}$ for FeCo-PA and FeCo-HDAHCI NPs, in good agreement with the TEM mean size. A thin oxide shell (ca. $1 \mathrm{~nm}$ ) is clearly observed on the HRTEM and high angle annular dark field image scanning TEM (HAADF-STEM, Figure S8). This shell is formed due to air-exposure during the sample introduction through air in the TEM. The freshly prepared particles are purely metallic, as evidenced by the XRD patterns performed under Ar atmosphere as further confirmed by advanced spectroscopies (see below). 


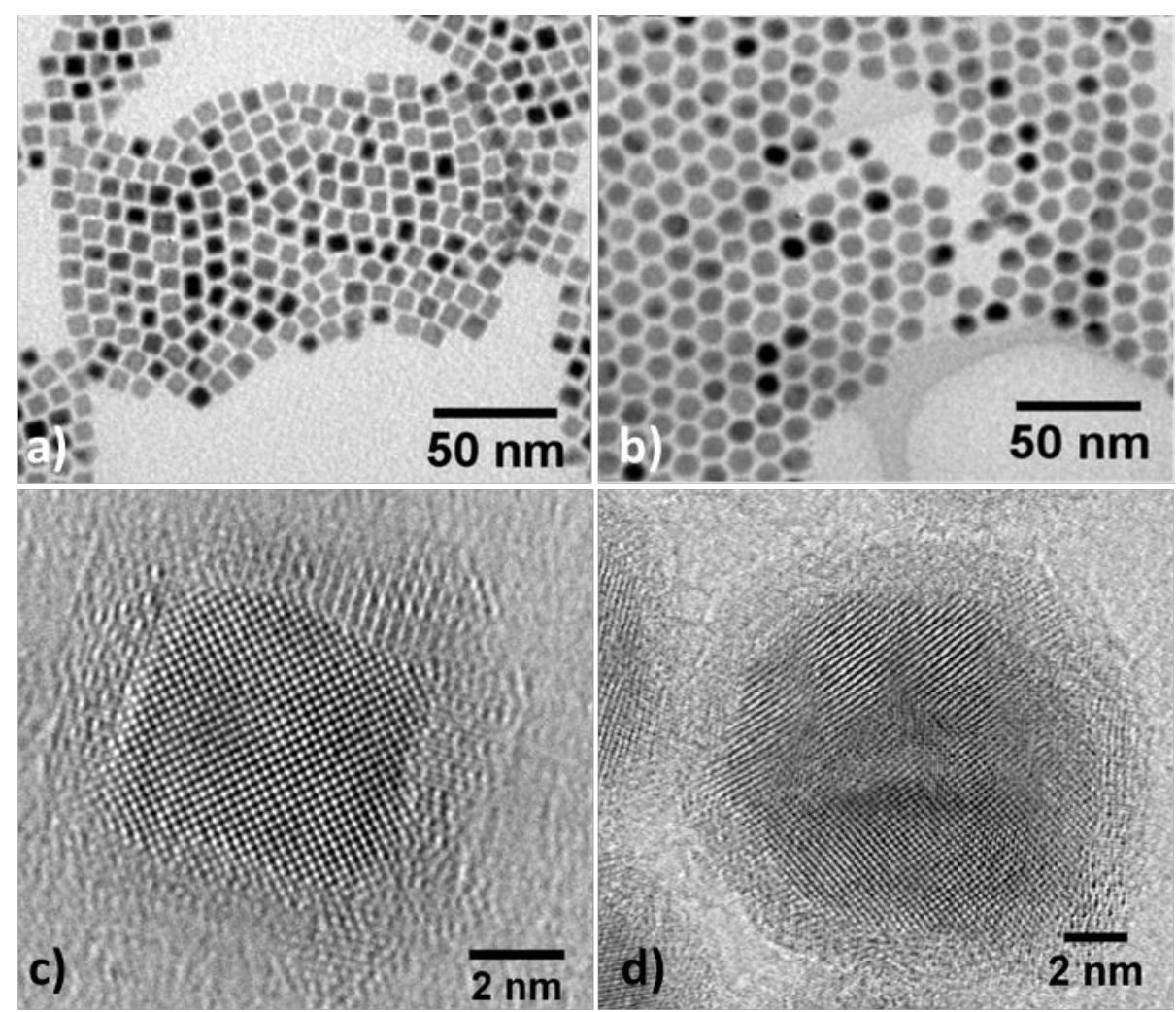

Figure 1: $a-b)$ Conventional transmission electron microscopy (TEM) micrographs. c-d) HRTEM images of FeCo nanoparticles prepared with PA (left column) or HDAHCl (right column).

Chemical analyses were performed on the final powder by inductively coupled plasma mass spectrometry (ICP-MS) revealing a global composition of $\mathrm{Fe}_{52} \mathrm{Co}_{48}$ for both type of NPs, very close to the equimolar ratio introduced. The local distribution of $\mathrm{Fe}$ and $\mathrm{Co}$ within the nanoparticles was further characterized by electron energy loss spectroscopy (EELS, Figures S8-9) and energy dispersive X-ray spectroscopy (EDX, Figure S10). STEM-EDX mapping revealed a local composition of $\mathrm{Fe}_{53} \mathrm{CO}_{47}$, in good agreement with the ICP-MS, revealing the homogeneity of the NP chemical composition. The distribution of the Fe and Co within individual NPs was further evidenced by probe-corrected STEM-EELS profile. While Co-rich core was always detected for FeCo-PA NPs (Figure S8), three different profiles were statistically identified in the FeCo-HDAHCl NPs, corresponding to Co-rich core, onion-like core and FeCo core (Figure S9).

The chemical order was studied at the atomic scale by combining ${ }^{57} \mathrm{Fe}$ Mössbauer spectroscopy and Zero field ${ }^{59} \mathrm{Co}$ ferromagnetic nuclear resonance (FNR i.e. nuclear magnetic 
resonance for ferromagnets) ${ }^{24}$ to probe respectively the Fe and Co environments without facing the interference of any oxidation artefacts ${ }^{25,26}$.
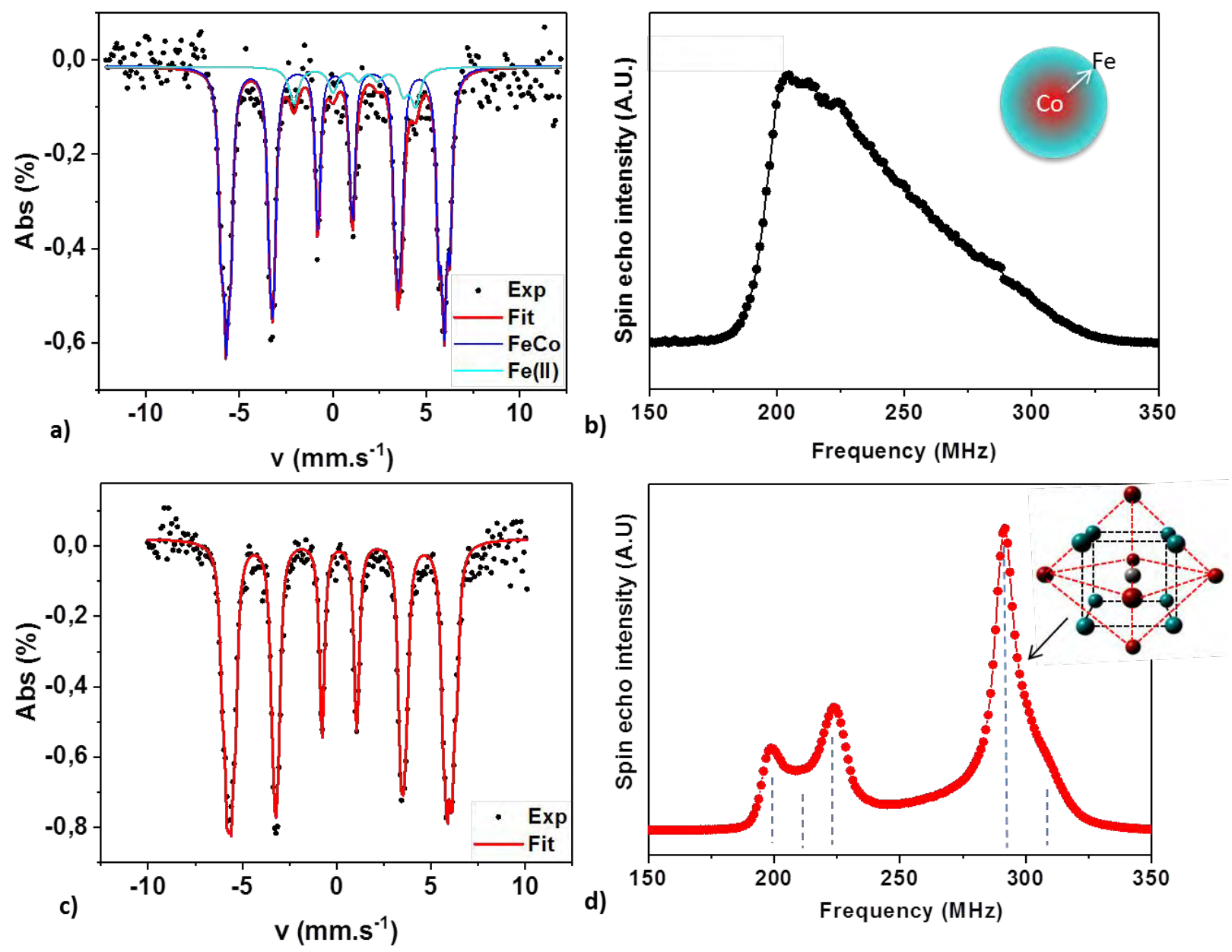

Figure 2: $a-c) 5 K^{57} \mathrm{Fe}$ Mossbauer spectrum and $\left.b-d\right){ }^{59} \mathrm{Co}$ Ferromagnetic Nuclear Resonance spectrum of the $a-b)$ FeCo-PA and c-d) FeCo-HDAHCl nanoparticles. As inset, schematic view of the gradient profile determined for PA NPs and B2 order for the HDAHCI NPs (Fe: blue, Co : red). Dashed lines are guides for the eyes for the different contributions on FeCo-HDAHCl FNR spectrum.

The Mössbauer signals recorded at $5 \mathrm{~K}$ and $300 \mathrm{~K}$ (see Figure S11) exhibit a sextet-like contribution, typical from FeCo alloys. ${ }^{25,27,28}$ The absence of any large hyperfine field contribution confirmed that after synthesis, the obtained particles are purely metallic and do not present any oxide shell. The fitting of the experimental spectra, reported in Table S1, evidenced broad hyperfine field distributions both for $\mathrm{HADHCl}$ and $\mathrm{PA}$ ligands. An additional contribution at low field in the case of PA ligands could be attributed to magnetically coupled Fe(II) molecular species as previously reported for pure Fe NPs, ${ }^{9}$ evidencing a slower reduction of the Fe species compared to the $\mathrm{HDAHCl}$ case. 
The ${ }^{59}$ Co FNR spectra clearly evidenced a major difference between the NPs obtained in the presence of PA and $\mathrm{HDAHCl}$ ligands. Indeed, a broad spin echo peak was observed in the frequency range from 180 to $320 \mathrm{MHz}$ for FeCo-PA NPs. The fitting of this peak (see figure S12) reveals the presence of a continuous concentration gradient from pure Co towards pure Fe across the NP radius (corresponding to 26 atomic layers). In the case of HDAHCl-NPs three main distinct peaks were observed at 200, 224 and $290 \mathrm{MHz}$ respectively and a shoulder at $211 \mathrm{MHz}$. The $200 \mathrm{MHz}$ peak can be attributed to a bcc Co metastable phase. Such a phase has been reported in a bcc epitaxial Fe/Co thin film structure, ${ }^{29}$ and could reveal the presence of NPs exhibiting a pure Co part close to a FeCo alloy. The $211 \mathrm{MHz}$ shoulder and the $224 \mathrm{MHz}$ contribution peak evidenced a structure composed of Co-rich bcc FeCo phase with 1st shell coordination polyhedra containing $7 \mathrm{Co}: 1 \mathrm{Fe}$ atoms and $6 \mathrm{Co}: 2 \mathrm{Fe}$ atoms respectively. An original short range order favoring even numbers of Fe atoms around Co has already been reported in CoFe alloy films. ${ }^{30}$ The simultaneous observation of the 200 and $224 \mathrm{MHz}$ peaks (with a much smaller $211 \mathrm{MHz}$ shoulder) might be the fingerprint of such unusual order. Finally, the major contribution in the spectra is at $290 \mathrm{MHz}$ and is characteristic of the chemically-ordered local environment known as $\mathrm{B2}$, with a $\mathrm{CsCl}$ structure (see inset figure $2 \mathrm{~d}$ ). ${ }^{31}$ Since the FNR spectrum reveals Co rich contributions in the 200-224 MHz range, the local B2 order has to be slightly enriched in Fe in order to preserve the FeCo stoichiometry. This is confirmed by the observation of the shoulder at $310 \mathrm{MHz}$ characteristic of a Fe enriched B2 order. To our knowledge, it is the first time that the shortrange B2 order is stabilized within NPs directly obtained after a liquid phase synthesis, thanks to the fine optimization of the precursor reactivity and the ligands used.

In-situ XRD study was performed during the thermal annealing of $\mathrm{FeCo}-\mathrm{HDAHCl}$ and FeCo-PA NPs (Figure 3 and S13) under a reducing atmosphere $\left(95 \% \mathrm{~N}_{2}, 5 \% \mathrm{H}_{2}\right)$ following a multi-step temperature profile up to $800^{\circ} \mathrm{C}$ (Figure S14). The use of a Co-source avoided the Fe atoms fluorescence and allowed the detection of the three superstructure reflections (100), (111) and (210) observed in the long-range crystalline B2 phase. ${ }^{32}$ The appearance of the B2 phase, which corresponds to a long-range chemical-ordered $\mathrm{CsCl}$-type structure, was characterized by the temperature evolution of the order parameter $S$, defined by : ${ }^{33,34}$

$$
S=\frac{r_{A}-x_{A}}{1-F_{A}} \quad e q(1)
$$


With $r_{A}$ the fraction of the site $A$ occupied by the right atom $A, x_{A}$ the atomic fraction of $A$ in the alloy $\left(\mathrm{x}_{\mathrm{Co}}=\mathrm{x}_{\mathrm{Fe}}=0.5\right)$ and $\mathrm{F}_{\mathrm{A}}$ the fraction of the site $\mathrm{A}\left(\mathrm{F}_{\mathrm{Co}}=\mathrm{F}_{\mathrm{Fe}}=0.5\right)$.

For a $\mathrm{Fe}_{50} \mathrm{CO}_{50}$ alloy, $\mathrm{S}$ values vary between $\mathrm{S}=0$ for a fully disordered alloy to $\mathrm{S}=1$ for a fully ordered B2 alloy. In the case of FeCo-HDAHCl NPs, a shoulder at the (100) peak is already observed at $300^{\circ} \mathrm{C}$, while the three characteristic peaks are clearly evidenced at $400^{\circ} \mathrm{C}$, leading to an order parameter $S=0.7$ (Figure 3c). The order parameter keeps on increasing, reaching $\mathrm{S}=0.76$ at $700^{\circ} \mathrm{C}$. Above this temperature, the order vanishes, as expected from the bulk phase diagram of FeCo alloys. After cooling down to room temperature, the long-range B2 phase was retrieved as evidenced by the order parameter $S=0.76$. In contrast for FeCoPA NPs, the partial ordering only appears at $500^{\circ} \mathrm{C}$ and was characterized by an order parameter of 0.55 (Figure S13). Such thermal behavior is in agreement with the previous report on the thermal ordering of disordered $30 \mathrm{~nm} \mathrm{Fe}_{50} \mathrm{CO}_{50}$ NPs prepared by polyol process. ${ }^{32}$ The fact that FeCo-HDAHCl NPs exhibit the B2 structure at lower temperature and with a better ordering factor than previously reported NPs and FeCo-PA NPs results from the presence of a short-range B2 order within as-prepared FeCo-HDAHCl NPs, as previously shown by Co FNR. The short-range B2 order region acted as seeds during the recrystallization process which was thermally activated, leading to a long-range chemical ordering under mild conditions $\left(300-400^{\circ} \mathrm{C}\right)$. 


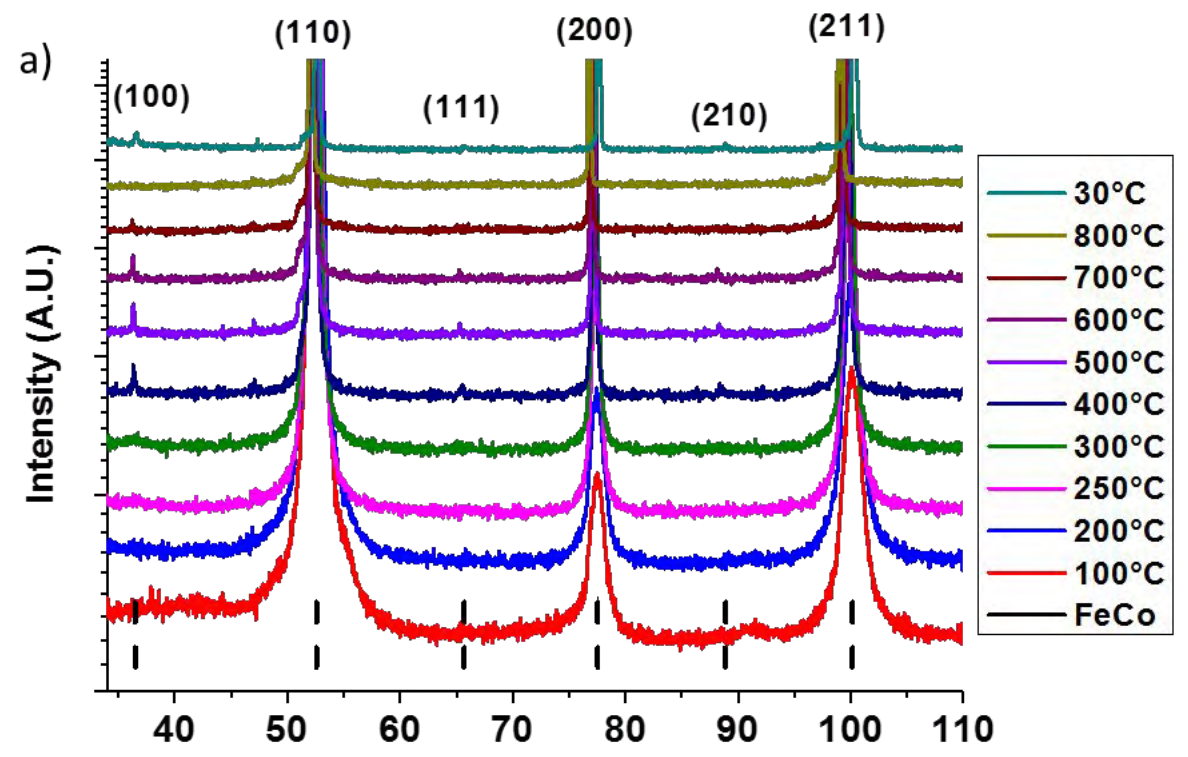

b)

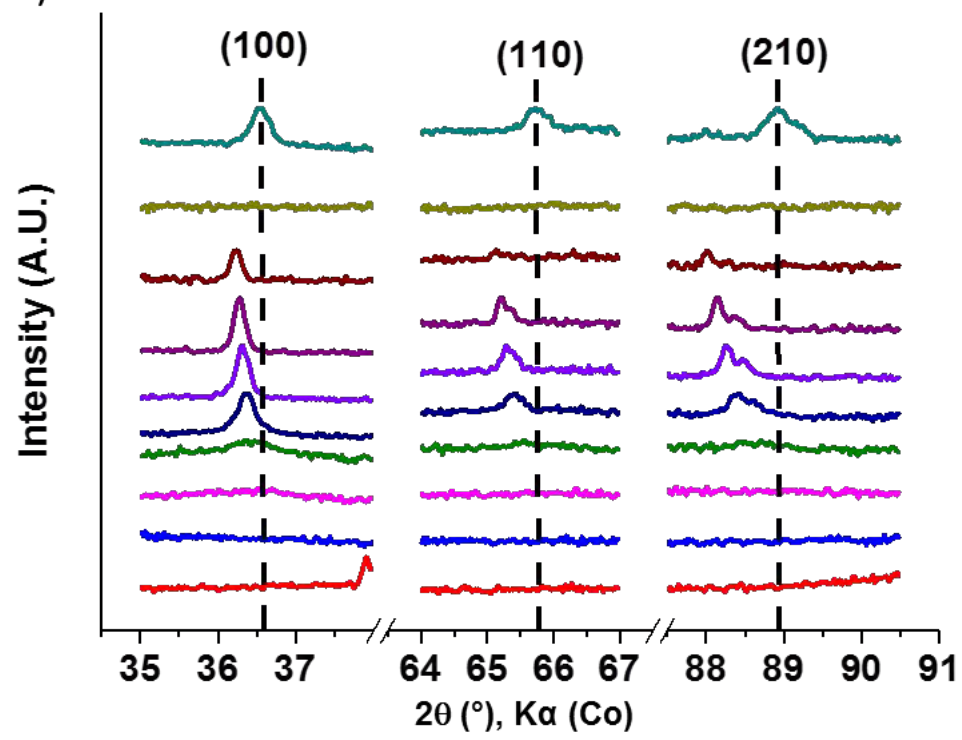

c)

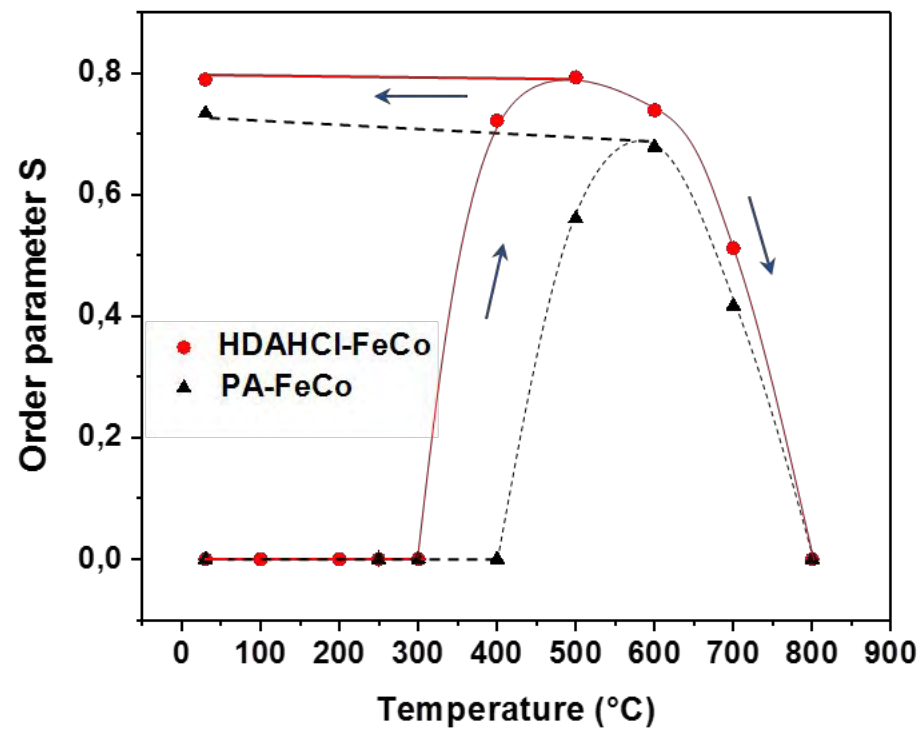


Figure 3: a) In-situ XRD recorded during thermal annealing of FeCo-HDAHCl NPs under reducing atmosphere. b) Enlarged view of the characteristic B2 superstructures peaks. c) Thermal evolution of the order parameter $S$ determined for $\mathrm{FeCo}-\mathrm{HDAHCl}$ (red dots) and FeCo-PA (black triangles) NPs. Red solid line, black dashed line and blue arrows are guide for the eyes following the temperature increase and the final cooling.

Both type of NPs exhibit a soft magnetic behavior at room temperature (Figure 4), with saturation magnetizations $\left(M_{S}\right)$ of 226 and $204 \mathrm{~A} \cdot \mathrm{m}^{2} \cdot \mathrm{kg}^{-1} \mathrm{FeCo}_{\text {for }} \mathrm{HDAHCl}$ and PA NPs respectively, very close to the expected bulk value $\left(\mathrm{Ms}_{\mathrm{Fe} 50 \mathrm{cc} 050}=235 \mathrm{~A} \cdot \mathrm{m}^{2} \cdot \mathrm{kg}^{-1} \mathrm{Feco}\right) \cdot{ }^{10}$ The low temperature curve (Figure S15) revealed no exchange bias indicating that the FeCo nanoparticles are not oxidized, in agreement with the Mössbauer spectroscopy.

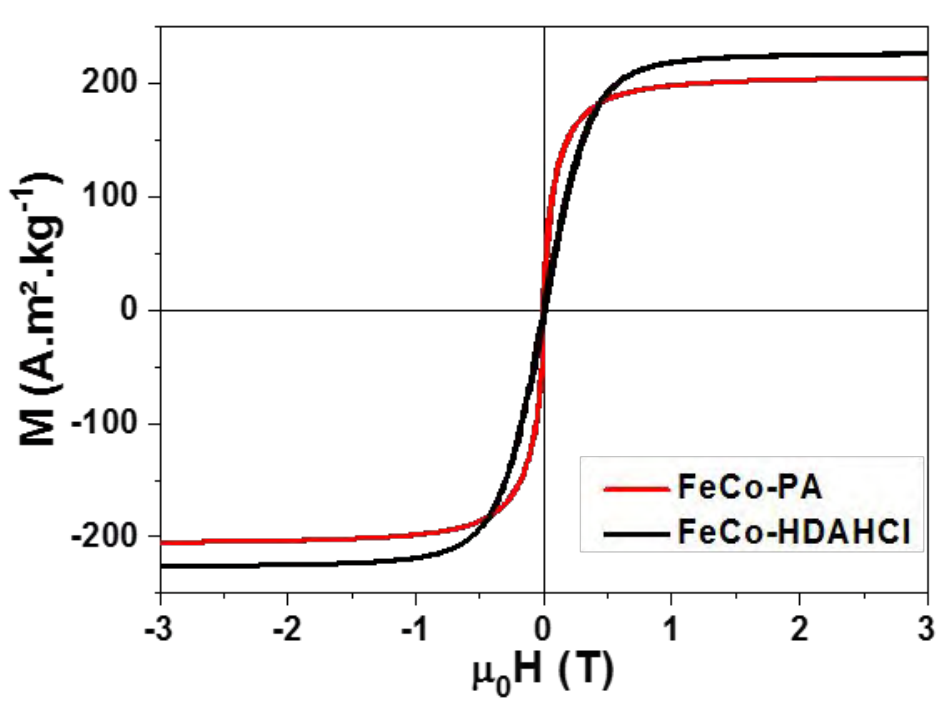

Figure 4: Magnetic hysteresis loop at 300K of the as-synthesized FeCo NPs prepared in presence of PA (black line) or HDAHCl ligands (red line).

The organometallic synthesis of FeCo NPs starting from two amide precursors yielded for the first time highly crystalline NPs with tunable sizes. In the presence of palmitic acid, carboxylate-containing precursors of $\mathrm{Co}$ and Fe which have different stabilities, lead to NPs exhibiting a concentration gradient. ${ }^{9,35}$ Thus, one can assume that the reduction followed a two-step process consisting in a first nucleation of Co nuclei, which then favored the decomposition of the more stable Fe-carboxylate precursor, leading to a fairly slow NP growth. On the contrary, in the presence of $\mathrm{HDAHCl}$, the intermediate chloride species 
formed seem to have a comparable reactivity, preventing the formation of a pure Co core. The concomitant co decomposition of the Fe and Co species lead for the first time to the synthesis of the chemically ordered short-range B2 structure. Therefore, a very fine tuning of the mild reaction conditions opens the route for the synthesis of magnetic alloys with controllable chemical structure and thus physical properties.

For the integration purposes, the net magnetic fraction into the composite materials being a key parameter, as-synthesized FeCo-HDAHCI NPs were preferred to FeCo-PA NPs since they exhibit a higher magnetization and a larger metallic content ( $80 \%$ and $50 \%$ respectively). These NPs were mixed with epoxy monomers and let to polymerize at room temperature to form ca. $250 \mu \mathrm{m}$ thick self-supporting composite pellets (Figures 5a inset and S16). The epoxy resin was chosen as a matrix for the FeCo composites since it advantageously combines electrical insulating characteristics, essential for high frequency applications, with an enhanced chemical resistance and efficient barrier properties to oxygen and moisture. ${ }^{36}$ The dispersion of the nanoparticles within the composite pellet cross section was characterized using SEM and TEM (Figures 5a-b). Thanks to a slow evaporation of the NP solvent, only few aggregates are observed, FeCo NPs being well dispersed along the whole thickness of the pellet. The loading of the composite could be reproducibly varied from 10 to $65 \%$ weight fraction of FeCo NPs, leading to a net magnetization under an applied field of 3T varying from 26 to $152 \mathrm{~A} \cdot \mathrm{m}^{2} \cdot \mathrm{kg}^{-1}$ (Figures 5c and S17, and Table S2). Assuming that the density of each components of the composite materials is similar to its bulk value, namely $\rho_{\mathrm{FeCo}}=8.3, \rho_{\text {ligands }}=0.9$ and $\rho_{\text {epoxy }}=1.1$, net magnetic volume fractions varying from 2 to $19 \%$ were determined. 
a)
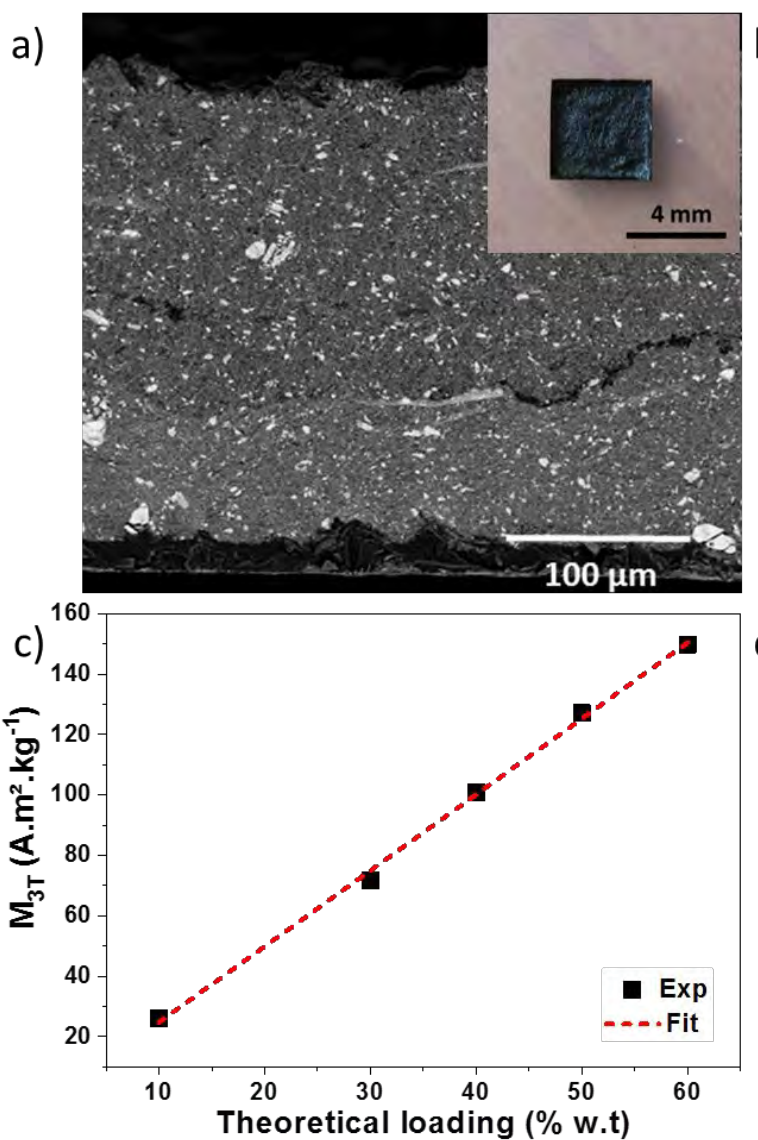

e)

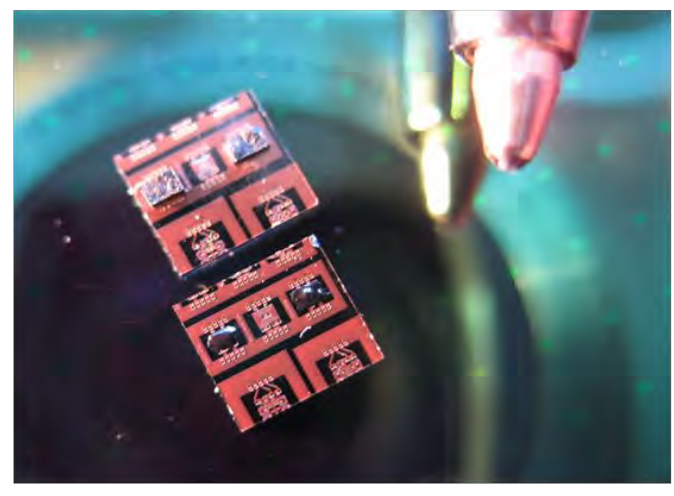

b)
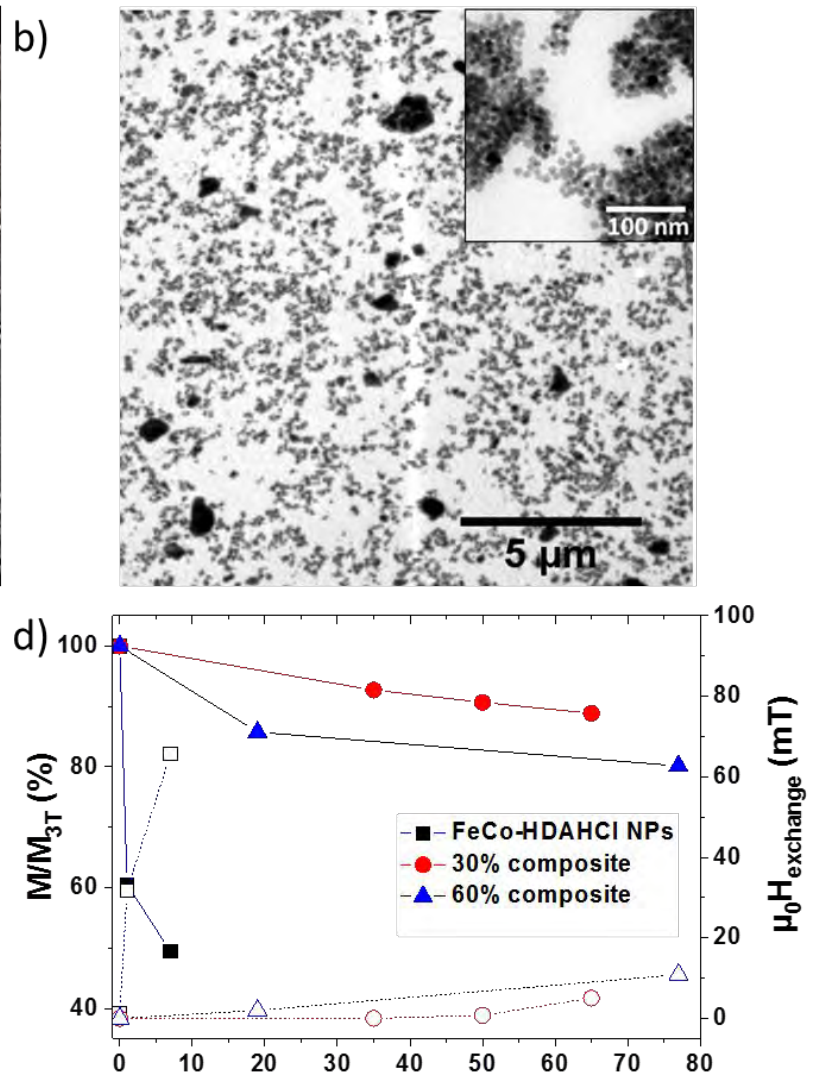

Time of air exposure (days)

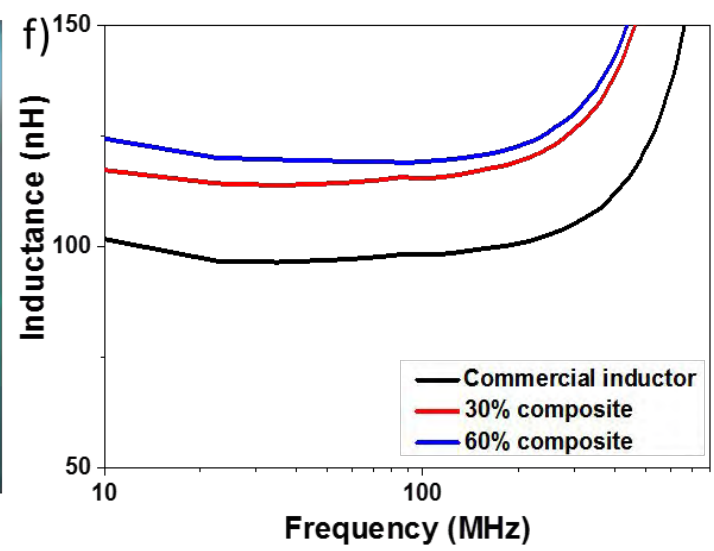

Figure 5: a) SEM image of the cross section of a 30\% in weight FeCo-HDAHCI NP loaded pellet. Due to the use of backscattered electrons, the metallic NPs appear as white dots while the epoxy appears grey, inset: optical image of the $4 \mathrm{~mm}$ squared pellet b) TEM image of an ultramicrotome slice of the epoxy composite. c) Evolution of the room temperature magnetization at $3 T\left(M_{3 T}\right)$ of the composites as a function of the targeted FeCo loading. $A$ linear fitting is superimposed as a guide for the eyes. d) Evolution of the relative $3 T$ magnetization (solid scatters and lines) and the exchange bias (open scatters and dashed lines) as a function of air exposure for pure FeCo-HDAHCl NPs (black) and the epoxy composite loaded with 30\% (circles) and 60\% (triangles) in weight FeCo-HDAHCl NPs. e) Picture of commercial silicon chip containing copper micro-inductors topped with FeCo rich epoxy composites of this study, f) evolution of the inductance as a function of frequency for the commercial inductor (black line) and a similar inductor covered with epoxy composite loaded with $30 \%$ (red line) and 60\% (blue line) in weight of FeCo-HDAHCI NPs. 
The magnetic properties were assessed as a function of time under air exposure to probe the protection against the oxidation of the epoxy matrix. The magnetization of pure FeCoHDAHCl NPs quickly diminished due to a strong oxidation once exposed to the air. The exchange bias, measured at low temperature increased up to $65 \mathrm{mT}$ after only 5 days. In the case of the epoxy-FeCo composite, 80 to $90 \%$ of the magnetization was retained after a 65 day air exposure for $60 \%$ and $30 \%$ loaded composite respectively. An exchange bias limited to 14 and $8 \mathrm{mT}$ respectively confirmed that the FeCo NPs are fairly well protected against the oxidation, the epoxy resin acting as an efficient air barrier. The resulting composite was successfully integrated on a commercial inductor-based filter using a pick and place approach. Enhancement of $18 \%$ and $27 \%$ of the inductance value at $100 \mathrm{MHz}$ were measured with the $30 \%$ and $60 \%$ loaded composite respectively compared to the air-core commercial inductor reference. Such increase represents an important step for the development of commercial planar inductors.

\section{Conclusion}

We report here a new chemical synthesis of monodisperse FeCo nanoparticles using the codecomposition of two metalloid amides under mild conditions without post treatment annealing. A fine tuning of the precursors reactivity was achieved thanks to the ligands used leading to two different NP type. For the first time the presence of the ordered B2 phase was evidenced in NPs prepared in presence of HDAHCl ligands, leading to magnetic properties comparable with the bulk ones $\left(\mathrm{Ms}=226 \mathrm{~A} \cdot \mathrm{m}^{2} \cdot \mathrm{kg}^{-1}\right)$. Optimized composite materials were prepared by mixing these NP with epoxy monomers. Composites with variable magnetic loadings were prepared and successfully integrated on inductor-based filters, leading to a $27 \%$ enhancement of the inductance value at $100 \mathrm{MHz}$. Although this enhancement remains modest this study evidences the possibility to use ex situ synthesized NPs for inductance improvement, opening the path for NP-based composites for advanced microelectronics applications after improvement of the magnetic volume fraction of the composite and of the device geometry by the full immersion of the inductors into the magnetic material.

\section{Acknowledgements:}


Lucien Datas and Jean-François Meunier are warmly thanked for high resolution TEM and Mössbauer spectroscopy measurements. This work was performed in the frame of TOURS 2015, project supported by the French «Programme de l'économie numérique des Investissements d'Avenir». We gratefully acknowledge the International Associated Laboratory (LIA)-M²OZART for financial support. Some of the HR-STEM and EELS studies were conducted at the Laboratorio de Microscopias Avanzadas, Instituto de Nanociencia de Aragon, Universidad de Zaragoza, Spain. R. A. gratefully acknowledges the support from the Spanish Ministry of Economy and Competitiveness (MINECO) through project MAT201679776-P (AEF/FEDER. UE). In IPCMS Strasbourg the work was supported by the CNRS LIA "NANOFUNC" and the LABEX NIE (No. ANR-11-LABX-0058_NIE).

\section{Experimental Section:}

\section{General synthesis}

Mesitylene (99\%), toluene (99\%), tetrahydrofuran (THF, 99\%) and pentane (99\%) were purchased from Merck, Acros, Carlo Erba and Fisher respectively. The solvents were purified on alumina desiccant (Pure Solv, Innovativ Technology) and degassed through three freezepump-thaw cycles. Hexadecylamine (HDA, 99\%) was purchased from Sigma-Aldrich. The two metallic precursors $\left(\mathrm{Fe}\left\{\mathrm{N}\left[\mathrm{Si}\left(\mathrm{CH}_{3}\right)_{3}\right]_{2}\right\}_{2}\right)_{2} \quad(\mathrm{FeNSi},>99.9 \%)$ and $\mathrm{Co}\left(\mathrm{N}\left(\mathrm{Si}\left(\mathrm{CH}_{3}\right)_{3}\right)_{2}\right)_{2}, \mathrm{THF}(\mathrm{CoNSi}$, $>99.9 \%$ ) were purchased from Nanomeps.

Synthesis of HDAHCl.

Hexadecylamonium chloride ( $\mathrm{HDAHCl})$ was synthesized following an adapted procedure described elsewhere ${ }^{[37]}$. Briefly, a solution of $10 \mathrm{mmol}$ of hexadecylamine (HDA) (2.415 g) in $400 \mathrm{~mL}$ of pentane was prepared in the glove box. An excess amount of $\mathrm{HCl}$ in diethylether $(14 \mathrm{~mL}, 1.0 \mathrm{M})$ was added under Ar atmosphere. The solution turned milky white immediately. The solution was stirred for $30 \mathrm{~min}$ then allowed to decant. After the removal 
of the supernatant, the resulting white concentrated solution was washed 2 times with pentane. The final solution was evaporating and $2,5 \mathrm{~g}(90 \%$ yield) of white powder $(\mathrm{HDA} \bullet \mathrm{HCl}$ ) was obtained.

Synthesis of FeCo nanoparticles.

* Preparation of the precursor solution

In a glove box, $150 \mathrm{mg}(0,4 \mathrm{mmol})$ of $\mathrm{Fe}\left(\mathrm{N}\left(\mathrm{Si}\left(\mathrm{CH}_{3}\right)_{3}\right)_{2}\right)_{2}$ and $180 \mathrm{mg}(0,4 \mathrm{mmol})$ of $\mathrm{Co}\left(\mathrm{N}\left(\mathrm{Si}\left(\mathrm{CH}_{3}\right)_{3}\right)_{2}\right)_{2}$, THF were separately dissolved in $2,5 \mathrm{ml}$ of mesitylene and then mixed leading to a green solution.

* Preparation of FeCo-PA NPs

$308 \mathrm{mg}(1,2 \mathrm{mmol})$ of PA and $386 \mathrm{mg}(1,6 \mathrm{mmol})$ of HDA were dissolved in $10 \mathrm{ml}$ and $5 \mathrm{ml}$ of mesitylene respectively. The PA solution was added onto the precursor mixture, which became blue. The resulting solution was magnetically stirred during $5 \mathrm{~min}$. The HDA solution was added, leading to a violet coloration which quickly vanished, the solution returning to blue after few seconds. The final $20 \mathrm{~mL}$ solution was transferred into a Fisher Porter bottle and pressurized under 3 bars of $\mathrm{H}_{2}$, the solution became darker. The reaction was heated at $150^{\circ} \mathrm{C}$ and let to react $48 \mathrm{~h}$ to allow the reduction of the stable Fe-carboxylate species formed in-situ.

\section{* Preparation of FeCo-HDAHCl NPs}

$334 \mathrm{mg}(1,2 \mathrm{mmol})$ of $\mathrm{HDAHCl}$ and $386 \mathrm{mg}(1,6 \mathrm{mmol})$ of HDA were dispersed in $10 \mathrm{ml}$ and 5 $\mathrm{ml}$ of mesitylene respectively. The precursor solution was added onto the $\mathrm{HDAHCl}$ one, which became brownish. The resulting solution was magnetically stirred during $5 \mathrm{~min}$, the solution turning to dark blue. Finally, the HDA solution is added. The final $20 \mathrm{~mL}$ solution is transferred into a Fisher Porter bottle and placed in a pre-heated oil bath at $150{ }^{\circ} \mathrm{C}$ for $1 \mathrm{~h}$ under Ar blanket to ensure the complete dissolution and homogenization of the reactants. The solution was then allowed to cool down at room temperature and further pressurized under 3 bars of $\mathrm{H}_{2}$. The reaction was heated again at $150^{\circ} \mathrm{C}$ for $24 \mathrm{~h}$ to allow the decomposition of the precursors.

* Purification of the NPs 
At the end of both type of reaction, the $\mathrm{H}_{2}$ atmosphere was released, a black solution was observed. The NPs were recovered by magnetically assisted precipitation, washed twice with toluene and twice with THF and finally dried, leading to a black powder kept in the glove box. Reaction yields were calculated from the final metallic weight content of the powder over the initial Fe and Co weight introduced. Yields of 23 and 66\% were obtained for FeCo$\mathrm{PA}$ and FeCo-HDAHCl NPs, respectively, due to partial weight losses during the purification steps and a non complete reduction of the Fe-carboxylate species as previously discussed.

\section{Preparation of the magnetic composite.}

In a glove box, $1,5 \mathrm{~mL}$ of THF is added on $50 \mathrm{mg}$ of powder. To ensure a proper dispersion, the solution was sonicated for $30 \mathrm{~min}$. To prepare a composite loaded with $30 \%$ of metal in weight, $50 \mathrm{mg}$ of the epoxy monomer (LY5052) and $19 \mathrm{mg}$ of the hardener (Aradur 5052) were then added. The resulting solution was further sonicated for $30 \mathrm{~min}$ and drop casted in a PDMS mold with 4 pitches of $4 \times 4 \times 0.5 \mathrm{~mm}^{3} .5 \mu \mathrm{L}$ drops were deposited in each pitch and the THF allowed evaporating. To obtain a sufficient thickness, the process was repeated 13 times. After a $24 \mathrm{~h}$ reticulation of the epoxy resin at room temperature, mechanically stable $\sim 250 \mu \mathrm{m}$ thick composite pellets were retrieved.

\section{Structural and chemical characterizations}

The chemical compositions were determined by chemical analysis using inductively coupled plasma mass spectrometry (ICP-MS, Pascher laboratory).

FeCo NPs were characterized by transmission electron microscopy (TEM), using a 100kV Jeol JEM 1011F and a JEM-ARM200F Cold FEG microscope with corrected probe. For sample preparation, the particles were redispersed in toluene and a drop of the dispersion was deposited on a carbon coated copper grid. The chemical profile measured by energy loss spectroscopy were acquired using the Scanning TEM mode with a probe size of 0,15 $\mathrm{nm}$ and steps of $0.477 \mathrm{~nm}$. Energy X-ray dispersive analysis were conducted routinely on a Scanning Electron Microscopy (SEM) JEOL 2100F equipped with a SSD Brucker detector to determine the relative FeCo content.

Spatial-resolved electron energy loss spectroscopy (EELS) measurements were performed on probe-corrected scanning transmission electron microscope (STEM) FEI Titan Low-Base 60- 
300 operating at $300 \mathrm{keV}$ (fitted with a X-FEG ${ }^{\circledR}$ gun and Cs-probe corrector (CESCOR from CEOS $\mathrm{GmbH}$ )). EEL spectra were recorded using the spectrum-imaging (SPIM in 2D or spectrum-line (SPLI) in 1D) mode ${ }^{[38]}$ in a Gatan GIF Tridiem ESR 865 spectrometer. The convergent semi-angle was of $25 \mathrm{mrad}$, the collection semi-angle was of $80 \mathrm{mrad}$ and the energy resolution $\sim 1.2 \mathrm{eV}$.

XRD measurements were performed on a PANalytical Empyrean diffractometer using Co-K $\alpha$ radiation and equipped with a linear Pixcel1D detector. In a glovebox, few mg of the powder was sealed between two kapton sheets to prevent any air exposure. The in-situ annealing measurements were performed in a sealed chamber XRK900 from Anton Paar under a reducing atmosphere $\left(1 \mathrm{bar}, 5 \% \mathrm{H}_{2}, 95 \% \mathrm{~N}_{2}\right.$ ). The powder patterns were measured at room temperature, $100^{\circ} \mathrm{C}, 200^{\circ} \mathrm{C}, 250^{\circ} \mathrm{C}, 300^{\circ} \mathrm{C}, 400^{\circ} \mathrm{C}, 500^{\circ} \mathrm{C}, 600^{\circ} \mathrm{C}, 700^{\circ} \mathrm{C}, 800^{\circ} \mathrm{C}$ then $30^{\circ} \mathrm{C}$ after cooling down at a rate of $c a .17^{\circ} \mathrm{C} / \mathrm{min}$ (Figure S12). For FeCO-PA NPs, the two first steps at $100^{\circ} \mathrm{C}$ and $200^{\circ} \mathrm{C}$ were skipped. At each step the sample was maintained for $1 \mathrm{~h}$ prior to the XRD acquisitions consisting of a $35-110^{\circ}$ acquisition in 20 minutes with $0.067^{\circ} . \mathrm{s}^{-1}$ as scan speed and a step of $0.052^{\circ}$, and three high-resolution acquisitions in the $35-38^{\circ}, 64-67^{\circ}$ and $88-91^{\circ}$ region with an accumulation of 24 minutes.

The rietveld refinement for the determination of the order parameter $\mathrm{S}$ was performed using MAUD software. ${ }^{[37]}$ the refinement involved the fit of the background polynomia, the scale factor, the profile parameters, the lattice parameters, one equivalent Debye-Waller factor and the occupancy rate of Co and Fe atoms on the special positions $(000)$ and $\left(\frac{111}{222}\right)$. In order to keep the stoichiometry, the atomic occupancy were restrained using the relations below :

$$
\begin{aligned}
& x_{C o}^{(000)}+x_{C o}^{\left(\frac{111}{222}\right)}=x_{F e}^{(000)}+x_{F e}^{\left(\frac{111}{222}\right)}=1 \\
& x_{C o}^{(000)}+x_{F e}^{(000)}=x_{C o}^{\left(\frac{111}{222}\right)}+x_{F e}^{\left(\frac{111}{222}\right)}=1
\end{aligned}
$$

With $x_{C o}^{(000)}$ corresponding to $r_{A}$ in eq(1). 
Mössbauer spectra were measured at $300 \mathrm{~K}$ and $5 \mathrm{~K}$ using a ${ }^{57} \mathrm{Co}$ source (WISSEL). Samples were prepared in the glovebox, and extreme care was taken to avoid oxidation during transfer to the apparatus. The analysis of Mossbauer spectra was performed by fitting the data by Lorentzian shape peaks and applying a least-squares method using Recoil software.

The ${ }^{59} \mathrm{Co}$ FNR experiments (NMR in ferromagnets) were performed at $2 \mathrm{~K}$ without any external static field in a home-made spectrometer. The integrated spin-echo intensity was recorded using a broadband un-tuned (high-pass design) pulsed FNR spectrometer with phase-sensitive detection and automated frequency scanning (pulse length: $5 \mu$, delay: 3 $\mu \mathrm{s})$. The frequency response of the setup is flat within $0,5 \mathrm{~dB}$. In order to prevent oxidation the samples were prepared in the glove box and sealed in regular NMR tubes with vacuum grease and epoxy resin.

\section{Physical characterizations}

Magnetic measurements were performed using a Quantum Design Physical Property Measurement System (PPMS) in the Vibrating Sample Magnetometer (VSM) configuration. For the FeCo NPs, $10 \mathrm{mg}$ of powder were introduced in a Teflon cap sealed by vacuum grease. For the composite, the pellet was directly mounted on a quartz sample holder. 3T magnetization cycles were recorded at $300 \mathrm{~K}$ and $5 \mathrm{~K}$ after a $3 \mathrm{~T}$ field cooling.

\section{Supporting Information Available}

Additional characterizations of FeCo-PA and FeCo-HDAHCI NPs : TEM images (as a function of acid concentration and reaction time) and the corresponding size distribution, Fe and Co elemental distribution determined by EELS (2D mapping and line profiles) and EDX, XRD pattern and $5 \mathrm{~K}$ magnetization curves. Mössbauer spectrum recorded at $300 \mathrm{~K}$ for FeCoHDAHCl NPs and the full set of parameters used for Mössbauer analysis. FNR spectrum and the corresponding fit for FeCo-PA NPs. Temperature profile applied during In situ XRD, and diffractograms obtained on FeCo-PA NPs.

Additional characterizations of 30 and $60 \%$ loaded composite : TEM images, 300K magnetization curves of $\mathrm{FeCo}-\mathrm{HDAHCl}$ and 30 and $60 \%$ loaded composite, and theoretical and experimental loading in weight percentage of the composite. 


\section{References}

[1] V. Korenivski, J. Magn. Magn. Mater. 2000, 215-216, 800-806.

[2] H. Yun, X. Liu, T. Paik, D. Palanisamy, J. Kim, W. D. Vogel, A. J. Viescas, J. Chen, G. C. Papaefthymiou, J. M. Kikkawa, et al., ACS Nano 2014, 8, 12323-12337.

[3] L. Wu, A. Mendoza-Garcia, Q. Li, S. Sun, Chem. Rev. 2016, 116, 10473-10512.

[4] S. Sun, Adv. Mater. 2006, 18, 393-403.

[5] N. Liakakos, T. Blon, C. Achkar, V. Vilar, B. Cormary, R. P. Tan, O. Benamara, G. Chaboussant, F. Ott, B. Warot-Fonrose, et al., Nano Lett. 2014, 14, 3481-3486.

[6] A. Bordet, L.-M. Lacroix, P.-F. Fazzini, J. Carrey, K. Soulantica, B. Chaudret, Angew. Chem. Int. Ed. 2016, 55, 15894-15898.

[7] L. Mohammed, H. G. Gomaa, D. Ragab, J. Zhu, Particuology 2017, 30, 1-14.

[8] L.-M. Lacroix, D. Ho, S. Sun, Curr. Top. Med. Chem. 2010, 10, 1184-1197.

[9] L.-M. Lacroix, S. Lachaize, A. Falqui, M. Respaud, B. Chaudret, J. Am. Chem. Soc. 2009, $131,549-557$.

[10] J. M. D. Coey, Magnetism and Magnetic Materials, Cambridge University Press, Cambridge, 2010.

[11] R. Ferrando, J. Jellinek, R. L. Johnston, Chem. Rev. 2008, 108, 845-910.

[12] T. Ogawa, H. Takano, H. Kura, M. Takahashi, J. Appl. Phys. 2012, 111, $07 B 533$.

[13] D. Kodama, K. Shinoda, K. Sato, Y. Konno, R. J. Joseyphus, K. Motomiya, H. Takahashi, T. Matsumoto, Y. Sato, K. Tohji, et al., Adv. Mater. 2006, 18, 3154-3159.

[14] F. Fiévet, S. Ammar-Merah, R. Brayner, F. Chau, M. Giraud, F. Mammeri, J. Peron, J.-Y. Piquemal, L. Sicard, G. Viau, Chem. Soc. Rev. 2018, 47, 5187-5233.

[15] Q. Yan, S. Li, E. Pang, Y. Wang, Mater. Lett. 2014, 120, 185-188.

[16] M. Y. Rafique, L. Pan, M. Zubair Iqbal, Q. Javed, H. Qiu, Rafi-ud-din, M. H. Farooq, Z. Guo, J. Alloys Compd. 2013, 550, 423-430.

[17] Y. Gu, Y. Cao, H. Chi, Q. Liang, Y. Zhang, Y. Sun, Int. J. Mol. Sci. 2013, 14, 14204-14213.

[18] X.-W. Wei, K.-L. Wu, G.-X. Zhu, Y.-J. Liu, W. Shi, X.-Z. Li, L.-L. Rong, L. Chen, F.-H. Wu, J. Alloys Compd. 2012, 539, 21-25.

[19] M. Abbas, M. N. Islam, B. P. Rao, K. E. Abou Aitah, C. Kim, Mater. Lett. 2015, 139, 161164.

[20] C. Desvaux, C. Amiens, P. Fejes, P. Renaud, M. Respaud, P. Lecante, E. Snoeck, B. Chaudret, Nat. Mater. 2005, 4, 750-753.

[21] C. Desvaux, F. Dumestre, C. Amiens, M. Respaud, P. Lecante, E. Snoeck, P. Fejes, P. Renaud, B. Chaudret, J. Mater. Chem. 2009, 19, 3268.

[22] C. Desvaux, P. Lecante, M. Respaud, B. Chaudret, J. Mater. Chem. 2010, 20, 103.

[23] A. Meffre, B. Mehdaoui, V. Kelsen, P. F. Fazzini, J. Carrey, S. Lachaize, M. Respaud, B. Chaudret, Nano Lett. 2012, 12, 4722-4728.

[24] P. Panissod, C. Meny, Appl. Magn. Reson. 2000, 19, 447-460.

[25] M. F. Casula, G. Concas, F. Congiu, A. Corrias, A. Falqui, G. Spano, J. Phys. Chem. B 2005, 109, 23888-23895.

[26] Y. Liu, J. Luo, Y. Shin, S. Moldovan, O. Ersen, A. Hébraud, G. Schlatter, C. Pham-Huu, C. Meny, Nat. Commun. 2016, 7, 11532.

[27] B. deMayo, J. Appl. Phys. 1970, 41, 1319.

[28] G. Concas, F. Congiu, G. Ennas, G. Piccaluga, G. Spano, J. Non-Cryst. Solids 2003, 330, 234-241.

[29] J. Dekoster, E. Jedryka, C. Meny, G. Langouche, EPL Europhys. Lett. 1993, 22, 433. 
[30] M. Wojcik, J. P. Jay, P. Panissod, E. Jedryka, J. Dekoster, G. Langouche, Z. Für Phys. B Condens. Matter n.d., 103, 5-12.

[31] J. P. Jay, M. Wójcik, P. Panissod, Z. Für Phys. B Condens. Matter 1996, 101, 471-486.

[32] G. B. Chon, K. Shinoda, S. Suzuki, B. Jeyadevan, Mater. Trans. 2010, 51, 707-711.

[33] M. Castiella, C. Gatel, J. F. Bobo, N. Ratel-Ramond, R. Tan, M. Respaud, M. J. Casanove, Mater. Res. Express 2015, 2, 086401.

[34] B. E. Warren, X-Ray Diffraction, Addison-Wesley, 1969.

[35] N. Liakakos, B. Cormary, X. Li, P. Lecante, M. Respaud, L. Maron, A. Falqui, A. Genovese, L. Vendier, S. Koïnis, et al., J. Am. Chem. Soc. 2012, 134, 17922-17931.

[36] F.-L. Jin, X. Li, S.-J. Park, J. Ind. Eng. Chem. 2015, 29, 1-11.

[37] K. Soulantica, A. Maisonnat, M.-C. Fromen, M.-J. Casanove, B. Chaudret, Angew. Chem. Int. Ed. 2003, 42, 1945-1949.

[38] R. Arenal, F. de la Peña, O. Stéphan, M. Walls, M. Tencé, A. Loiseau, C. Colliex, Ultramicroscopy 2008, 109, 32-38. 